\title{
EEG-based identification of evidence accumulation stages in decision making
}

\author{
Hermine S. Berberyan ${ }^{1}$, Leendert van Maanen ${ }^{2}$, Hedderik van Rijn $^{3}$, \& Jelmer P. Borst ${ }^{1}$ \\ ${ }^{1}$ Bernoulli Institute, University of Groningen, Groningen, the Netherlands \\ ${ }^{2}$ Department of Experimental Psychology, Utrecht University, Utrecht, the Netherlands \\ ${ }^{3}$ Department of Experimental Psychology, University of Groningen, Groningen, the Netherlands
}

Corresponding author:

Hermine S. Berberyan, h.berberyan@rug.nl

ORCID:

Hermine S. Berberyan 0000-0002-3889-5933

Leendert van Maanen 0000-0001-9120-1075

Hedderik van Rijn 0000-0002-0461-9850

Jelmer P. Borst 0000-0002-4493-8223

This is a preprint. A peer reviewed publication is Berberyan, H., van Maanen, L., van Rijn, H., \&

Borst, J. P. (in press). EEG-based identification of evidence accumulation stages in decision making.

Journal of Cognitive Neuroscience. https://doi.org/10.1162/jocn_a_01663 


\begin{abstract}
Dating back to the $19^{\text {th }}$ century, the discovery of processing stages has been of great interest to researchers in cognitive science. The goal of this article is to demonstrate the validity of a recently developed method, hidden semi-Markov model multivariate pattern analysis (HsMM-MVPA), for discovering stages directly from EEG data, in contrast to classical RT-based methods. To test the validity of stages discovered with the HsMM-MVPA method, we applied it to two relatively simple tasks where the interpretation of processing stages is straightforward. In these visual discrimination EEG experiments, perceptual processing and decision difficulty were manipulated. The HsMM-MVPA analysis revealed that participants progressed through five cognitive processing stages while performing these tasks. The brain activation of one of those stages was dependent on perceptual processing, while the brain activation and the duration of two other stages was dependent on decision difficulty. Additionally, evidence accumulation models (EAMs) were used to assess to what extent the results of HsMM-MVPA are comparable to standard RT-based methods. Consistent with the HsMM-MVPA results, EAMs showed that non-decision time varied with perceptual difficulty and drift rate with decision difficulty. Moreover, non-decision and decision time of the EAMs correlated highly with the first two and the last three stages of the HsMM-MVPA analysis, respectively, indicating that the HsMMMVPA analysis gives a more detailed description of stages discovered with this more classical method. The results demonstrate that cognitive stages can be robustly inferred with the HsMM-MVPA analysis.
\end{abstract}

Keywords: Hidden semi-Markov models, multivariate pattern analysis, EEG, evidence accumulation models, stage discovery. 


\section{Introduction}

A central tenet of theories in cognitive neuroscience is the existence of processing stages. Following the seminal work of Donders (1868|1969) and Sternberg (1969), stages are typically identified based on behavioral responses. While these methods are easy to apply and provide insight in the relative duration of different processing stages, it has proven difficult to identify the exact duration and temporal onsets of individual stages (e.g., Henson, 2011; Posner, 2005). Here, we demonstrate the validity of a recently proposed method, hidden semi-Markov model multivariate pattern analysis (HsMM-MVPA), as a method for discovering processing stages using neural data (EEG/MEG).

The HsMM-MVPA method was introduced by Anderson et al. (2016) to overcome the limitations of conventional methods based on behavioral data. The main advantage of HsMM-MVPA is that it allows parsing a cognitive task into processing stages based on the many EEG/MEG samples within a trial, unlike one RT measure per trial. For example, when it was applied to EEG data from the classic Sternberg task, five stages were identified: 1) pre-attention, 2) encoding, 3) memory retrieval, 4) decision, and 5) response (Anderson, Zhang, Borst, \& Walsh, 2016). The HsMM-MVPA method has been proved to be a versatile method for detecting processing stages in a range of tasks (Anderson et al., 2018; Borst \& Anderson, 2015; Portoles, Borst, \& van Vugt, 2018; Walsh, Gunzelmann, \& Anderson, 2017; Zhang, Borst, Kass, \& Anderson, 2017; Zhang, van Vugt, Borst, \& Anderson, 2018; Zhang, Walsh, \& Anderson, 2017, 2018). At the same time, the interpretation of discovered stages remains a challenge. In particular, when dealing with more complex tasks that involve a longer sequence of cognitive processes, it becomes difficult to find support for a specific cognitive interpretation.

The main goal of the current paper is to demonstrate the validity of the HsMM-MVPA analysis as a method for discovering processing stages that can be linked to a specific cognitive interpretation. Because we do not have access to ground truth - we cannot observe cognitive processes directly - we will use experimental manipulations to provide a proxy of ground truth. In this way, robust conclusions on discovered processes can be drawn. Additionally, the HsMM-MVPA analysis will be complemented by evidence accumulation models (EAMs) that represent a traditional, well-established approach to modeling decision making (Evans \& Wagenmakers, 2020). Analogous to Donders' and Sternberg's 
methods, EAMs are applied to RTs; however, they provide a more formal account of behavioral data. In doing so, EAMs are able to separate cognitive processes underlying the RT distribution, in particular, decision processes from non-decision processes (e.g., Bode et al 2018; Jepma et al, 2012).

With this goal in mind, we designed two EEG experiments in which participants were asked to perform a simple visual discrimination task. Each experiment consisted of two conditions where different visual stimuli were used to manipulate perceptual processing. Between experiments, we maintained the same stimuli and response options but made the decision more difficult. Thus, between conditions, perceptual stages should be affected, while between experiments a decision stage(s) is expected to have a different duration. Before applying the HsMM-MVPA method, we will confirm the successful operationalization of these assumptions by using EAMs.

To summarize: 1) we conducted two experiments in which we manipulated perceptual processing and decision difficulty, 2) we quantified the duration of decision and non-decision components utilizing EAMs, 3) we applied the HsMM-MVPA method to discover the underlying processing stages, 4) we correlated the results of the EAMs with those of HsMM-MVPA models. Overall, this set of analyses confirmed the effectiveness of HsMM-MVPA as a versatile method for stage discovery and resulted in a definition of simple stages that can be used to interpret more complex tasks. In the remainder of this introduction, we will describe the main assumptions underlying the application of the HsMM-MVPA method to EEG data and explain the use of EAMs.

\section{HsMM-MVPA analysis}

The HsMM-MVPA method decomposes EEG data into processing stages. The method is based on the assumption that any cognitive event - the start of a processing stage - is accompanied by a negative or a positive peak across different brain regions. This assumption is shared between the two main theories explaining the generation of ERPs: the classical theory and the synchronized oscillations theory. According to classical theory, phasic bursts of activity are generated when cognitive events occur (Schroeder et al., 1995; Shah et al., 2004). In this framework, background EEG is regarded as noise. The synchronized oscillations theory opposes this view and instead proposes that such peaks result from 
synchronization in a certain frequency band, or phase resetting, that is triggered by the event (Klimesch, Sauseng, \& Hanslmayr, 2007; Makeig et al., 2002). Although these theories suggest different causes on ERP generation, simulation studies revealed no difference in activity generated by phasic bursts and phase resetting: both theories predict significant positive or negative deflections at the start of a new cognitive process (Yeung, Bogacz, Holroyd, \& Cohen, 2004).

To identify the onset of cognitive processes, the HsMM-MVPA analysis searches for such negative or positive deflections across the scalp and in time. These so-called bumps mark the transition from one processing stage to another. It is assumed that bumps are separated by flats that have a variable duration; together with the bumps, they comprise stages in a cognitive task. To identify the optimal number of bumps to account for EEG data, separate HsMM-MVPA models are fitted that assume different numbers of bumps. By comparing the goodness-of-fit of these models, the optimal number is derived, providing the temporal locations and scalp topologies of the bumps, and the distributions of durations of the flats (see Methods for details).

Figure 1 illustrates how the EEG signal from three different trials is modelled with the HsMMMVPA analysis. The algorithm searches for bumps that represent the onset of a cognitive stage and flats that separate these bumps. In these flats, the EEG signal is described by a sinusoidal noise around 0 . The goal is to identify the topology and the temporal location of each bump on each trial. As there is variability in the duration of cognitive processes and the associated EEG signal on each trial, the algorithm allows for bumps to occur at different time points in each trial (i.e., a perceptual stage will have a different duration on each trial, jittering the onset of the subsequent bump). To account for this, we analyze data at the single-trial level, while taking into account all trials of all participants simultaneously. Because the method assumes that the cognitive processes are the same on each trial, the topologies of the bumps are kept constant across trials (depicted by the red arrows in Figure 1). However, as the length of each process can vary per trial, the durations of the flats are variable for all subjects and trials, described by gamma distributions (depicted by the blue arrows). The HsMM-MVPA model illustrated in Figure 1 contains five bumps, each marking the onset of a cognitive stage. As the first stage starts with the stimulus presentation, this model represents six stages in total; the end of the last stage is defined by the subject's response. 


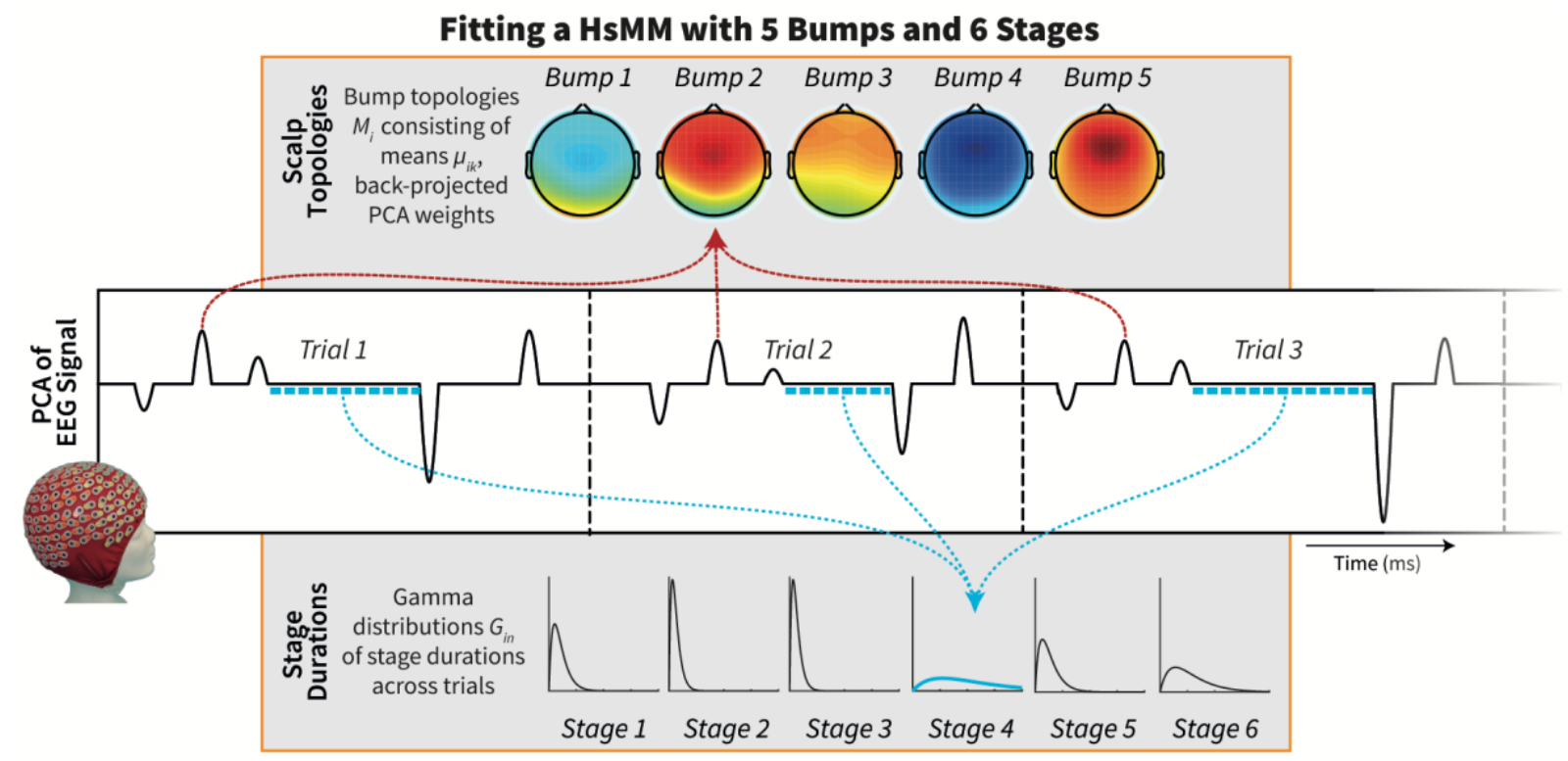

Figure 1. An illustration of the HsMM-MVPA method applied to EEG data from three trials. The resulting model contains 5 bumps and 6 stages. Red dashed arrows indicate the location of the Bump 2 on each trial, whereas blue dashed arrows indicate variability in the duration of Stage 4, that represents the process terminated with Bump 4.

\section{Modelling decision tasks with EAMs}

To decompose reaction times into underlying cognitive components, EAMs such as driftdiffusion model (DDM; Ratcliff, 1978) are often used. The DDM is designed for modelling data from two-choice experiments and is well established in cognitive science (Ratcliff \& McKoon, 2008). The core idea of this method is that participants gather evidence for certain decisions over time before they execute a response. Because this evidence accumulation process is noisy, reaction times across trials vary, and participants sometimes make mistakes. This model has been successfully applied to a wide variety of tasks (e.g. Gomez, Ratcliff, \& Perea, 2007; Milosavljevic, Malmaud, Huth, Koch, \& Rangel, 2010; Smith \& Ratcliff, 2004).

Because the current tasks required only very simple decisions, participants' responses were mostly correct. As a standard DDM model is not well suited for modelling tasks with very few errors (Royce Anders, Alario, \& Maanen, 2016) we used the shifted Wald model instead (Heathcote, 2004; Matzke \& Wagenmakers, 2009). This model allows modelling a decision process which is most likely terminated by the correct response option. In Figure 2 an example of a shifted Wald model is shown. The red line represents the process of evidence accumulation with an average drift rate $v$. This 
accumulation process continues until a threshold $B$ is reached, and the response is executed. The decision time, therefore, depends on both the drift rate $v$ and the threshold value $B$. Non-decision time $(t O)$ accounts for non-decision processes such as encoding, pre-attention and motor preparation. Together with decision time, non-decision time comprises observed reaction times.

The parameters of a Wald model can be interpreted concerning their relation to cognitive processing. The drift rate value has been attributed to decision difficulty, with lower values corresponding to harder decisions (Basten, Biele, Heekeren, \& Fiebach, 2010; Mulder, van Maanen, \& Forstmann, 2014). The threshold value is typically associated with response caution (e.g., Boehm, Van Maanen, Forstmann, \& Van Rijn, 2014; Bogacz, Wagenmakers, Forstmann, \& Nieuwenhuis, 2010; van Maanen et al., 2019). Finally, the value of the non-decision component has been linked to enhanced attention due to stimulus anticipation (Jepma, Wagenmakers, \& Nieuwenhuis, 2012) and "stimulus quality" (Bode et al., 2018).

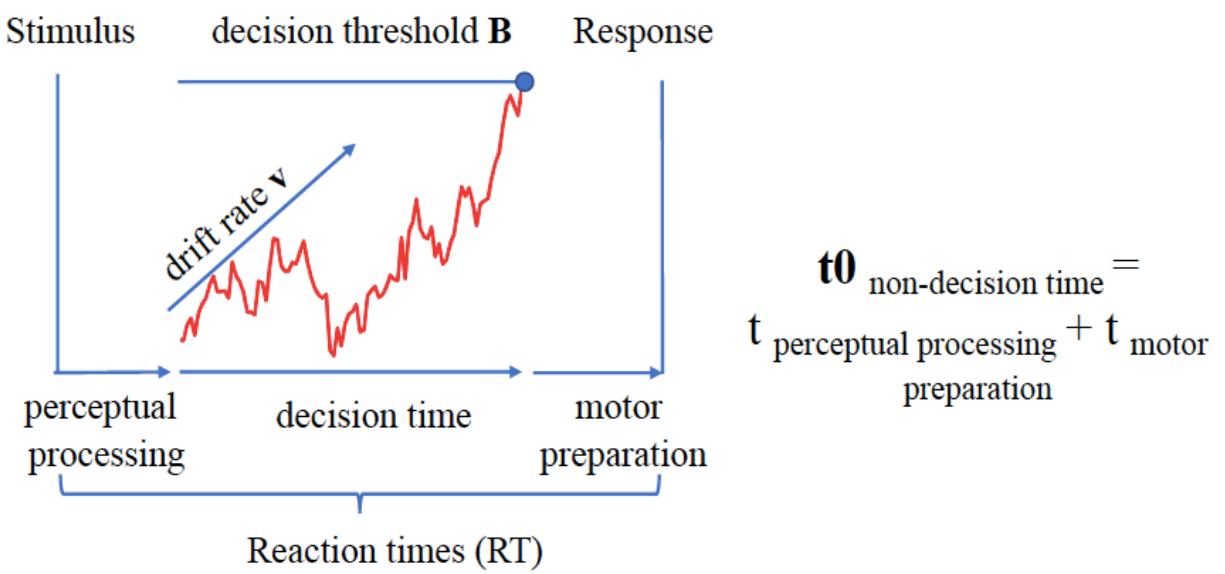

Figure 2. A visual representation of a shifted Wald model. The figure illustrates example data of a participant who accumulates evidence in a trial. This noisy accumulation process is represented with the red line with a mean drift rate $v$ and is terminated with a decision boundary, or threshold, $B$. The time that is not related to the decision process such as perceptual processing and motor preparation is represented as $t 0$, namely, non-decision time.

We will now describe two EEG experiments across which we varied perceptual processing and decision difficulty. Both experiments consisted of two conditions where different types of stimuli were used to manipulate perceptual processing. Between experiments, we used the same stimuli but adapted the task to vary decision difficulty. Overall, assuming that HsMM-MVPA is a versatile method for discovering cognitive stages in a task, we hypothesized that: 1) the topological distributions of HsMMMVPA-identified perceptual stage(s) will differ by stimulus type, 2) the duration of HsMM-MVPA 
decision stage(s) and the topology will be different when we manipulate task difficulty, 3) these differences should be consistent with non-decision time and drift rate values in corresponding Wald models.

\section{Methods}

\section{Participants}

In Experiment 1, thirty participants performed a simple visual discrimination task. Data of five participants were excluded (three due to the problems in EEG recording, one for excessive noise in the EEG, one for not following task instructions), resulting in a final set of 25 participants (13 females, mean age $=24.72, \mathrm{SD}=4.49$, range 18 to 33 years $)$.

In Experiment 2, thirty participants performed a more difficult visual discrimination task. Data of five participants were excluded from the analysis (three due to a programming error, one for major eye movement contamination that could not be captured with independent component analysis, one for EEG malfunction), resulting in a final set of 25 participants ( 16 females, mean age $=23.92, \mathrm{SD}=4.8$, range 18 to 36 years).

Participants in both experiments were students of the University of Groningen or the Hanze University of Applied Sciences in Groningen, The Netherlands. All participants were right-handed, had normal or corrected-to-normal vision, no history of cognitive impairment and normal color vision as assessed by the EnChroma Color Blindness Test (EnChroma, Inc. of Berkeley, $\mathrm{CA}^{1}$ ). Before the experiments, participants gave written informed consent to the experimental procedures, as approved by The Research Ethics Review Committee (CETO) of the Faculty of Arts at University of Groningen (reference number 62838174 for Experiment 1, 64834780 for Experiment 2), in accordance with the Declaration of Helsinki. Participants received monetary compensation of 8 euros.

\section{Task design}

\footnotetext{
${ }^{1}$ https://enchroma.com/pages/color-blindness-test
} 
In both experiments, participants were presented with geometric shapes or with character strings. Their task was to discriminate between different stimuli by using one of the two randomly assigned response keys ('M' or 'N'). Based on the Stimulus Type, the conditions are labelled Shapes or Characters. As we varied the Decision Difficulty between the two experiments, we will refer to Experiment 1 as Easy and Experiment 2 as Difficult. We will, therefore, refer to the Shapes condition of Experiment 1 as the Easy Shapes condition and the Characters condition of Experiment 1 as the Easy Characters condition. In line with that, the two conditions of Experiment 2 will be further referred to as Difficult Shapes and Difficult Characters.

The Easy Shapes condition was presented in two blocks. Four shapes (circle, triangle, square, rhombus) of four colors (red - \#ff0000, green - \#55aa00, yellow - \#ffff00, blue - \#0000ff) were used as stimuli. Red-green and blue-yellow colors were chosen in line with the opponent process theory where they are considered the highest contrasting pairs (Goldstein, Humphreys, Shiffrar, \& Yost, 2008; Hurvich \& Jameson, 1957). In Block 1, two shapes of two colors were randomly selected from the set, and participants were instructed to respond based on the shape of the stimulus and to ignore the color. In Block 2, the other two shapes and colors were used, and the task was to respond based on the color of the stimulus and ignore the shape. We will collapse over those two blocks in our analyses (the results of these blocks were also analyzed separately; however, no differences were found).

The Easy Characters condition was presented in one block. In this condition, participants were instructed to respond whether the characters were letters or numbers. The stimuli consisted of four random upper-case letters of the Latin alphabet (e.g., BDRN, or AENJ) or four random digits from 0 to 9 (e.g., 3476, or 8168)

The Difficult Shapes condition was presented in two blocks. In this condition, participants were presented with the same stimuli as in the Easy Shapes condition, however, the task was more difficult: they were instructed to respond by considering both shape and color of the objects. For example, if in Block 1 the stimuli were red or green triangles or circles, the participant was instructed to press ' $\mathrm{M}$ ' when a red circle or a green triangle was presented, and ' $\mathrm{N}$ ' for the other two stimuli (Figure 3). Thus, both features - shape and color - had to be taken into account. For Block 2, the task was the same, only different shapes and colors were used. 
The Difficult Characters condition was presented in one block. In this condition, participants were presented with the same stimuli as in the Easy Characters condition and were instructed to discriminate between consonants, vowels, odd, and even numbers. An example of this condition is illustrated in Figure 3 where the participant was instructed to press ' $\mathrm{M}$ ' for even numbers and consonants and ' $\mathrm{N}$ ' for odd numbers and vowels. To diminish the possible effects of interference we excluded the presentation of 'Q', 'Y', 'O' from letters and '0' from numbers.

To summarize, we designed Shapes and Characters conditions where we used Stimulus Type to manipulate perceptual processing and Easy and Difficult conditions where we manipulated Decision Difficulty.

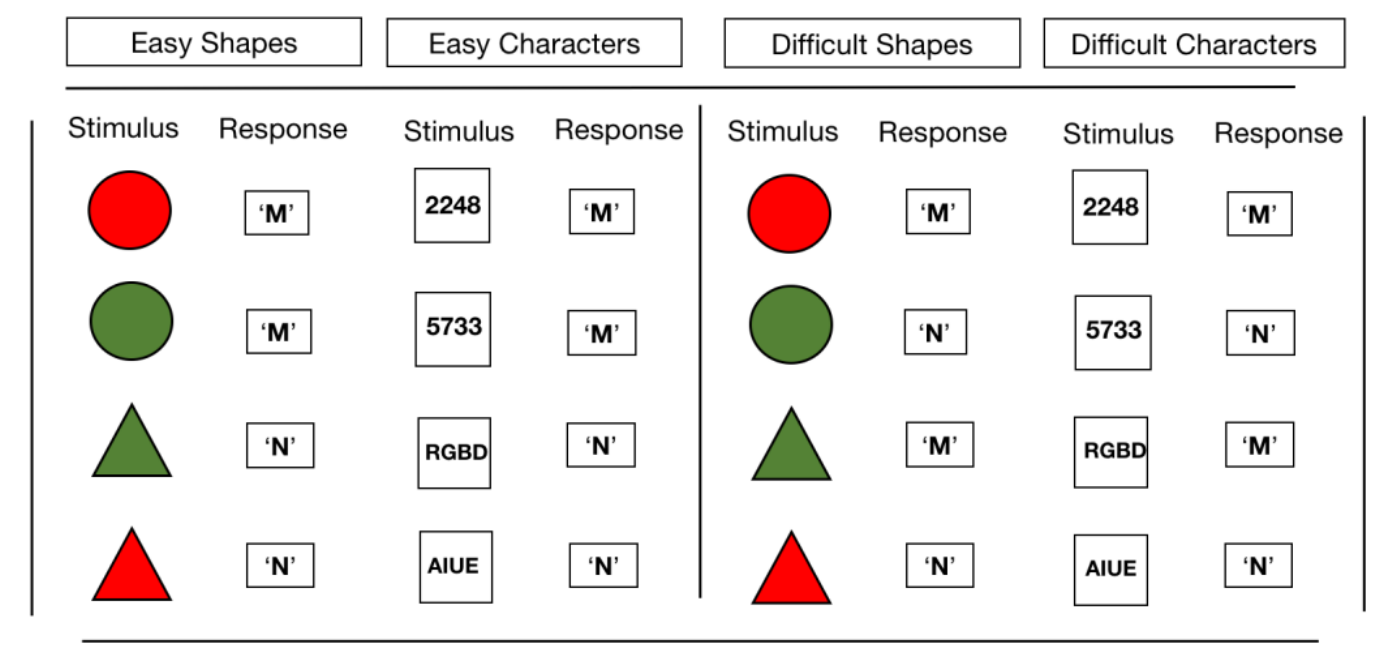

Figure 3. Response mapping in all conditions. In Easy Shapes, participants were asked to discriminate between two shapes of different colors (the color should be ignored) or based on two different colors of the shape (then the shape should be ignored). In Easy Characters, participants were asked to discriminate between two characters - a letter or a number. In Difficult conditions, the same stimuli were used while the Decision Difficulty was manipulated.

\section{Procedure}

Participants were seated in front of a 21.5 -inch screen with a resolution of $1366 \times 768$ pixels. The Easy conditions contained 480 trials: three blocks consisting of 20 practice and 140 main trials. The Difficult conditions contained 456 trials: three blocks consisting of 12 practice and 140 main trials. The presentation of the blocks was counterbalanced across participants.

Each block started with instructions, directly followed by practice trials. After the experimenter ensured the task was sufficiently understood, the experimental trials commenced. Each trial started with 
a fixation dot, presented for a duration sampled from a uniform distribution (from 1500 to $2250 \mathrm{~ms}$ ), followed by the presentation of the stimulus which remained on the screen until one of the two response keys ('N' or ' $M$ ') was pressed, or the response deadline was reached. In the Easy conditions, the deadline was set to $3000 \mathrm{~ms}$ and in the Difficult conditions, it was increased to $5000 \mathrm{~ms}$ to reflect the increased difficulty of the task. Feedback was provided for $500 \mathrm{~ms}$ ('Correct', 'Incorrect', or 'Late'). Between blocks, participants were given the option to take a short break. Each block took around 10 minutes. In total, data acquisition for both Easy and Difficult conditions lasted one hour, including EEG set up and instructions.

\section{Behavioral analysis}

For the behavioral analysis, we removed practice trials and incorrect trials. For each of the four conditions (Easy Shapes, Easy Characters, Difficult Shapes, Difficult Characters) and each participant, we then excluded trials that deviated more than 2 standard deviations from the mean reaction times of the participant and the condition (this preprocessing pipeline was used for all consecutive analyses, the qualitative results are identical when the 'outliers' are kept in the data; we used this rather strict outlier regiment to stay in line with previous HsMM-MVPA analyses).

To statistically evaluate reaction times and accuracy, linear mixed-effects models (LME models) were constructed (Bates \& DebRoy, 2004). The evaluation of models was done with a forward stepwise fitting routine: we started with simple models where only one predictor was included (Stimulus Type or Decision Difficulty), then the models with both predictors were evaluated. Lastly, the estimation terminated with the full model which included the interaction between Stimulus Type and Decision Difficulty. The lmerTest $\mathrm{R}$ package was used to obtain $p$-values for fixed effects based on Satterthwaite's method (Kuznetsova, Brockhoff, \& Christensen, 2018). Not only fixed effects but also random effects can potentially lead to statistical errors. To prevent the latter, the forward fitting procedure was separately applied to random effects. Thus, we determined the maximum random-effects structure allowed by the data (Barr, Levy, Scheepers, \& Tily, 2013; Bates, Kliegl, Vasishth, \& Baayen, 2015). 


\section{Evidence accumulation modelling}

Only the reaction times for correct responses were modelled because the error rates for all conditions and all participants were on average lower than 5\%. The model parameters were estimated using differential evolution Markov chain Monte Carlo algorithm (Sherri, Boulkaibet, Marwala, \& Friswell, 2019; Turner, Sederberg, Brown, \& Steyvers, 2013) as implemented in the Dynamic Models of Choice (DMC) software (Heathcote et al., 2019). A prior probability distribution for each of the parameters was specified as a truncated normal distribution. The convergence of samples was assessed with Gelman and Rubin's potential scale reduction factor (Gelman \& Rubin, 1992). To reduce the autocorrelation between different MCMC samples, thinning was applied.

We hypothesized that different conditions might lead to differences in the parameters of evidence accumulation models. In order to test this assumption, we estimated all possible models where the parameters drift rate $(v)$, threshold $(B)$ and non-decision time $(t 0)$ differed by Stimulus Type, separately for the Easy and Difficult conditions. The estimation started with the simplest model, the intercept only model, and terminated with the most complex model, where all parameters were assumed to differ per condition. Further, for each model, the Watanabe-Akaike information criterion was computed (Vehtari, Gelman, \& Gabry, 2015), and WAIC values were derived for each subject. We then calculated WAIC weights analogous to AIC weights' computation procedure (Wagenmakers \& Farrell, 2004) to allow for a more straightforward interpretation of WAIC differences in the estimated models.

\section{EEG recording and preprocessing}

The EEG was recorded from 32 positions using active Ag-AgCI electrodes (Biosemi Active Two system) digitized with a sampling rate of $512 \mathrm{~Hz}$. The electrodes were placed using the international 1020 system layout including two "ground" channels - Common Mode Sense (CMS) and Driven Right Leg (DRL). Two horizontal and two vertical electrodes were used to measure eye movements and blinks. Data were post-hoc referenced to the average of the mastoids. Scalp impedance for each electrode was kept under $20 \mathrm{k} \Omega$ for all except four participants, for whom it was kept under $30 \mathrm{k} \Omega$. 
For EEG preprocessing and analysis, the open-source toolbox EEGLAB (Delorme \& Makeig, 2004) was used along with custom-made scripts in MATLAB (The MathWorks, Inc). EEG data were passed through a high-pass filter of $1 \mathrm{~Hz}$ and a low-pass filter of $40 \mathrm{~Hz}$. Next, data were downsampled to 256 Hz. Manual artifact rejection was performed on continuous data by visual inspection of the data and rejection of data portions containing noise. On average, $3.7 \%$ of the data were removed due to artifacts. For six participants, one or two noisy channels were removed. Furthermore, data were decomposed with independent component analysis (ICA) to remove eye blinks and muscle artifacts (using EEGLAB's runica function, which is based on a logistic infomax algorithm, Bell \& Sejnowski, 1995). Typically, one or two components were identified to account for eye artifacts or muscle movements and were subsequently subtracted from the data. The channels that were removed were topographically reconstructed using spherical spline interpolation.

\section{Preprocessing for HsMM-MVPA}

For HsMM-MVPA analysis, additional preprocessing procedures were applied. First, the data was down-sampled to $100 \mathrm{~Hz}$ for computational trackability. Next, the data was epoched on a trial-by-trial basis relative to the onset of the presentation of the stimuli and consecutive response. That is, the analysis is performed on all data points of all trials between stimulus and response. The trials that were marked as outliers in the behavioral analysis were excluded. Next, a baseline from $400 \mathrm{~ms}$ preceding the stimulus appearance was computed and subtracted. Only complete trials were subjected to the analysis (incomplete trials appeared due to artifact rejection). A covariance matrix was computed for each trial and subject separately (Cohen, 2014; Portoles et al., 2018). Further, we performed principal component analysis (PCA) and the first 10 PC components accounting for $94.8 \%$ of the variance were retained. Finally, the data were normalized by calculating z-scores.

\section{HsMM-MVPA analysis}

The HsMM-MVPA analysis was applied to discover cognitive stages. A Hidden Markov model is a type of stochastic model that connects a sequence of hidden states to a sequence of corresponding 
observations. While the Hidden Markov model assumes that one observation corresponds to one state, in the Hidden semi-Markov model, several observations can be produced during a state and each of these states, therefore, has a variable duration (Yu, 2010). In the current application of HsMM-MVPA analysis, principal components extracted from the EEG signal act as observations, and the analysis aims to find cognitive stages that are hidden. To identify the onsets of these stages we search for bumps positive or negative deflections with a duration of $50 \mathrm{~ms}$. This duration was chosen as it produces robust results even if the actual bumps are slightly shorter or longer (Anderson et al., 2016). The process that is indicated by a bump continues within a flat; the mean amplitude of the signal during the "flat" is equal to zero. Together with bumps flats comprise cognitive stages. The duration of these flats was modelled with a gamma distribution with a shape parameter of 2 . The placement of $n$ bumps results in $n+1$ flats due to the first stage starting with a flat.

For Easy and Difficult conditions, different HsMM-MVPA models will be constructed. The model estimation starts with a single-bump model and increases to the number of bumps $n_{\max }$ which is the maximum number of bumps (each taking $50 \mathrm{~ms}$ ) that fit in the shortest observed reaction time. During the estimation of an HsMM-MVPA model, two parameters of the hidden states are obtained: 1) the amplitudes of the bumps that mark the onsets of cognitive stages and 2) the scale parameter of a gamma distribution that describes the stage durations (the shape parameter is fixed at 2). These parameters are estimated in a way that maximizes the match between the EEG data and the model for all trials with a standard Expectation-Maximization (EM) algorithm.

The fitting process starts with defining initial amplitudes for the bumps and gamma distributions for stage durations. As the convergence of the EM algorithm is sensitive to the choice of a starting point $(\mathrm{Wu}, 1983)$ and might end up in a local maximum, one option is to use different random values for bump amplitudes and compare their outputs (e.g., Portoles et al., 2018). Here we take another approach based on the work by Zhang, Walsh, Anderson (2018). We firstly fitted separate HSMM-MVPA models for each condition with the maximum number of bumps possible $\left(n_{\max }\right)$ and obtained the bump amplitudes and gamma distributions. Next, these parameters were used for models with fewer bumps $\left(n_{\max }-1\right)$ where we iteratively left out each of the bumps of $n_{\max }$. Then, all different $n_{\max }-1$ models were compared and the model with the best fit was selected. This process was repeated until a model with just a single bump 
$\left(n_{1}\right)$ was fitted. The idea was to find all potential bump topologies while avoiding local maxima. The amplitudes of the bumps obtained in this way have been further used as inputs for subsequent HsMMMVPA models.

The log-likelihood of HsMM-MVPA models tends to increase when more bumps are fitted, as there are more parameters to fit the data. To avoid overfitting, we applied a leave-one-out cross-validation (LOOCV) procedure. We estimated the HsMM-MVPA model on all subjects but one and then tested the fit of this model on the left-out subject, effectively separating training and testing of the models. This was repeated for all participants. Finally, we tested for how many subjects the log-likelihoods of the models with $n+1$ bumps increased compared to an $n$-bump model using a sign test. In this way, we evaluated whether a model with one additional bump outperformed the previous model for a sufficiently large number of participants, and thus whether the additional complexity of the model was warranted. As a result, the optimal number of bumps in each condition was obtained.

For more details, a mathematical description, and code of the HsMM-MVPA analysis we refer to Anderson et al. (2016).

\section{Results}

\section{Behavioral results}

Average error rates and reaction times (RT) along with within-subject standard errors (Morey, 2008) were computed for each condition and are shown in Figure 4. The proportion of errors in all conditions was found to be low (not exceeding $5 \%$ ). To quantify the differences in behavioral performance for both Stimulus Type and Decision Difficulty, we constructed LMEs. Using a stepwise fitting procedure, we ended up with the same fixed and random effects structure for RT and accuracy ( $p$ $<0.001$ for RT and $p<0.05$ for accuracy): Stimulus Type, Decision Difficulty and their interaction as fixed effects, Participant as a random intercept, Stimulus Type and Trial as random slopes. 

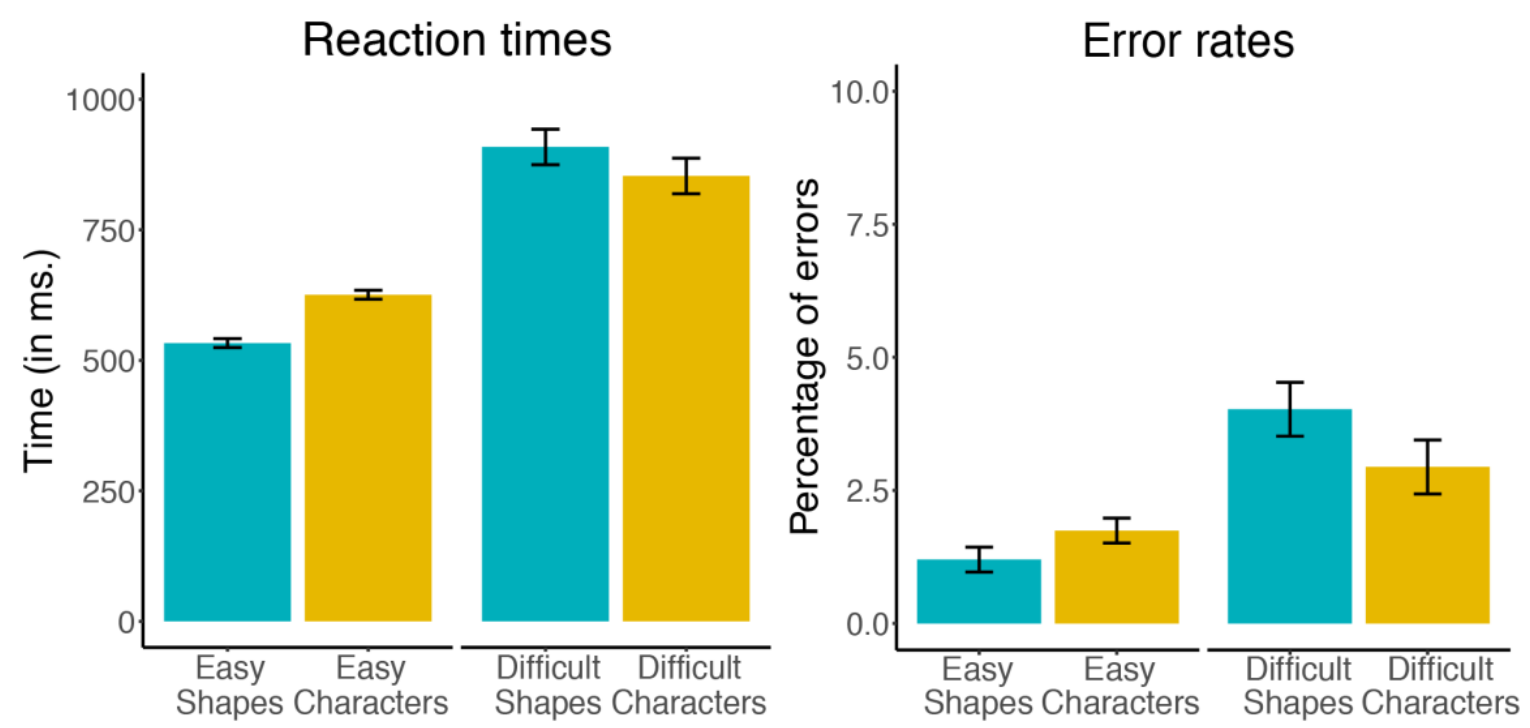

Figure 4. Mean reaction times and error rates with within-subject standard errors for each condition.

First, reaction times (RT) were plotted against a theoretical normal distribution and as they were found to be right-skewed, a logarithmic transformation was applied. Next, an LME model with log (RT) as a dependent variable was fitted. For the Easy conditions, Stimulus Type reached significance, indicating that participants were faster when discriminating between Easy Shapes than Easy Characters. For Difficult Conditions, Figure 4 suggests the inverse effect of Stimulus Type but it did not reach significance. For both Shapes and Characters, Decision Difficulty had a significant effect on reaction times, namely, RTs increased when the same stimuli were presented but the decision was more difficult (for statistics, see Table 1). Additionally, for all conditions, the interaction between Stimulus Type and Decision Difficulty was significant.

For accuracy as a dependent variable, a binomial LME model was fitted. For the Shapes condition, Decision Difficulty had a significant effect, but did not reach significance for the Characters condition. Additionally, for Difficult Conditions, Stimulus type had a significant effect on accuracy. For all conditions, the interaction between Stimulus Type and Decision Difficulty was significant.

To summarize, Decision Difficulty had the largest effect: it led to significantly longer reaction times for both Stimulus Types. Additionally, participants answered slower to Characters than to Shapes, but only when the decision was easy.

Table 1. The results of LME models for $\log (\mathrm{RT})$ and accuracy as dependent variables 


\begin{tabular}{|c|c|c|c|c|c|c|}
\hline \multirow[b]{3}{*}{$\begin{array}{l}\text { Reference: Easy } \\
\text { Shapes }\end{array}$} & \multicolumn{6}{|c|}{ Response variable } \\
\hline & \multicolumn{3}{|c|}{$\log (R T)$} & \multicolumn{3}{|c|}{ Accuracy } \\
\hline & Estimate & $\mathrm{t}$ value & p-value & Estimate & $\mathrm{z}$ value & p-value \\
\hline Intercept & 6.25 & 168.73 & $<0.001 * *$ & 4.78 & 22.28 & $<0.001 * *$ \\
\hline Stimulus Type & 0.14 & 3.88 & $<0.001 * *$ & -0.27 & -1 & 0.31 \\
\hline Decision Difficulty & 0.47 & 8.99 & $<0.001 * *$ & -1.27 & -4.63 & $<0.001 * *$ \\
\hline $\begin{array}{l}\text { Stimulus Type } * \\
\text { Decision Difficulty }\end{array}$ & -0.18 & -3.51 & $<0.001 * *$ & 0.82 & 2.47 & $<0.05^{*}$ \\
\hline \multicolumn{7}{|l|}{$\begin{array}{l}\text { Reference: Difficult } \\
\text { Characters }\end{array}$} \\
\hline Intercept & 6.68 & 143.34 & $<0.001 * *$ & 4.06 & 15.2 & $<0.001 * *$ \\
\hline Stimulus Type & 0.04 & 1.08 & 0.28 & -0.55 & -2.35 & $<0.05^{*}$ \\
\hline Decision Difficulty & -0.28 & -4.29 & $<0.001 * *$ & 0.44 & 1.19 & 0.23 \\
\hline
\end{tabular}

\section{Evidence accumulation results}

To identify how these differences in RTs were caused by decision and non-decision components we fitted Shifted Wald models to RTs. Various models - starting from the intercept only model to the most complex model where drift rate $(v)$, non-decision time $(t O)$ and threshold $(B)$ could differ - were compared with regard to WAIC weights. These weights represent the probability that a particular model is the 'true' model (i.e., the model that generated the data) under the assumption that the 'true' model is in the set of compared models (Wagenmakers \& Farrell, 2004). The quantified WAIC weights showed that there was no clear winning model across participants. To account for individual variability in the fitted models, we applied model averaging (Hoeting, Madigan, Raftery, \& Volinsky, 1999; Miletić \& van Maanen, 2019). For each subject, the estimated values from the models were weighted by the corresponding WAIC weights. Then, the average over all subjects was calculated per parameter $(v, t 0$, $B)$. The resulting weighted values are shown in Figure 5. 


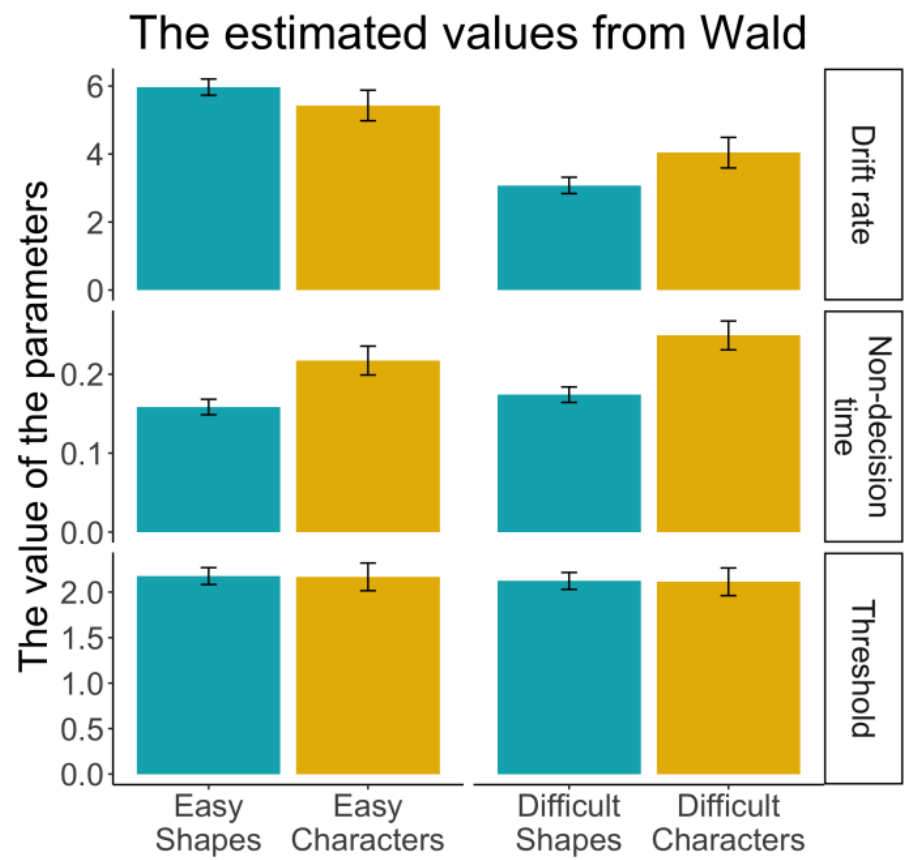

Figure 5. The average weighted values (weighted by the corresponding WAIC weights) are presented for each condition and for each of the Wald model parameters: drift rate, non-decision time and threshold.

To test whether these values differed per Stimulus Type and Decision Difficulty, separate LME models were constructed with Parameter $(v, t O, B)$ as the dependent variable and Participant as a random effect. The model estimation was performed following a forward stepwise fitting routine: it started with the simple models (Stimulus Type or Decision Difficulty as the only predictor) and terminated with the full models (main effects of Stimulus Type and Decision Difficulty and their interaction as predictors). To compare these models, we computed Bayes factors (BF) with the BayesFactor package (Morey, Rouder, \& Jamil, 2015). BF represents relative evidence in favor of null and alternative hypotheses provided by the data (Kass \& Raftery, 1995). In terms of model comparison, reported BFs indicate whether the data favors a model with an effect over a model with no effect, the intercept-only model.

For the drift rate, the BFs provided strong evidence for the model with Decision Difficulty (see Table 2 for statistics). This model outperformed all other models: vs. model with Stimulus Type, BF = $65762.5( \pm 0.03 \%)$; vs. 'main-effects model' of Stimulus Type and Decision Difficulty, $\mathrm{BF}=4.23$ $( \pm 0.9 \%)$; vs. 'full model' with main effects of Stimulus Type and Decision Difficulty and interaction, $\mathrm{BF}=3.59( \pm 1.11 \%)$. For the threshold parameter, BFs provided evidence against models with Stimulus Type and Decision Difficulty. For the non-decision time, BFs provided strong evidence for the model 
with Stimulus Type. This model outperformed all the other models except for the 'main-effects model': vs. model with Decision Difficulty, $\mathrm{BF}=6056.83( \pm 0 \%)$; vs. 'main-effects model', $\mathrm{BF}=1.16( \pm 9.4 \%)$; vs. 'full model', $\mathrm{BF}=3.78( \pm 4.16 \%)$. Although both models- the model with Stimulus Type and 'main effects model'- describe the data well, we were inclined to proceed with the model with Stimulus Type as it has the highest BF value compared to the intercept-only model and is the more parsimonious model.

To summarize, Shifted Wald models were fitted to the RT data from all conditions. Consistent with our hypothesis, we found evidence that Decision Difficulty affected the drift rate of the decisionmaking process, and that Stimulus Type affected the non-decision time.

Table 2. Bayes factors for the estimated Wald parameters (drift rate, threshold and non-decision time). Reported Bayes factors indicate relative fit by comparing these models to an intercept-only model. Asterisks $\left(^{*}\right)$ indicate the models with the highest BFs.

\begin{tabular}{|c|c|c|c|}
\hline & \multicolumn{3}{|c|}{ Response variable } \\
\hline Predictors & Drift rate $(v)$ & Threshold $(B)$ & Non-decision time $(t 0)$ \\
\hline Stimulus Type & $0.23 \pm 0.03 \%$ & $0.21 \pm 0.03 \%$ & $3791.48 \pm 0 \% *$ \\
\hline Decision Difficulty & $15227.53 \pm 0 \% *$ & $0.23 \pm 0.03 \%$ & $0.63 \pm 0 \%$ \\
\hline $\begin{array}{c}\text { main-effects model } \\
(\text { Stimulus Type + } \\
\text { Decision Difficulty) }\end{array}$ & $3595.3 \pm 0.9 \%$ & $0.05 \pm 1.86 \%$ & $3259.01 \pm 9.4 \%$ \\
\hline $\begin{array}{c}\text { 'full model' } \\
\text { (Stimulus Type } * \\
\text { Decision Difficulty) }\end{array}$ & $4245.34 \pm 1.11 \%$ & $0.01 \pm 8.82 \%$ & $1004.02 \pm 4.16 \%$ \\
\hline
\end{tabular}

\section{HsMM-MVPA results}

To gain more insight into the processing stages that comprise decision and non-decision processes, the HsMM-MVPA analysis was applied to all conditions separately to find the optimal number of stages. For all conditions, a model with four bumps and five stages accounted best for the data. Next, to test whether the discovered cognitive stages differed per Stimulus Type, for Easy and Difficult conditions, a range of HsMM-MVPA models were constructed in which bumps and stage durations were shared in different ways. The model estimation was performed with a forward stepwise fitting routine and included: a general model where we hypothesized that scalp topologies and stage durations for both 
conditions are the same; models where we varied each consecutive bump between conditions; and finally, completely separate models for the two conditions. The model fits were compared for each participant, and a model was preferred when the fit improved for a significant number of participants compared to a simpler model. Table 3 lists the results.

For the Easy conditions, the model where Bump 2 varied per Stimulus Type had the highest average loglikelihood over participants, except for the completely separate models. As a cognitive stage in an HsMM-MVPA model is represented in both bump topology and stage duration, the duration of the consecutive stage (Stage 3) was also varied. The resulting model in which we varied both Bump 2 and the duration of Stage 3 significantly outperformed all other combined models and was not outperformed by any other model. While this was also the case for completely separate models for each condition, these models require more parameters. We, therefore, decided to proceed with the more parsimonious model, in which only Bump 2 and Stage 3 were different per Stimulus Type.

For the Difficult conditions, we also estimated models where each bump was allowed to be different between conditions. However, none of these models outperformed the general model (Table 3). Because we observed a difference in duration of Stage 4 for the different Stimulus Types in the initial models, we complemented the analysis with a model where Stage 4 was varied. However, this model also did not outperform the general model.

The topographical representations and stage onsets of the final models are presented in Figure 6, the average duration of these stages and the standard errors are presented in Figure 7.

Finally, to investigate the effect of Decision Difficulty, the discovered HsMM-MVPA stages were compared between Easy and Difficult conditions. The durations of the resulting stages were visually inspected (Figure 8), and the stages that seemed to be different for Decision Difficulty - Stage 4 and Stage 5 - were subjected to statistical analysis. Thus, an LME model with Stage durations as a dependent variable, Decision Difficulty as a predictor and Participant as a random intercept was fitted. To assess the relative fit of these models, BFs were computed with the BayesFactor package (Morey, 2008). BFs provided strong evidence for the effect of Decision Difficulty for Stage $4\left(1.9 \times 10^{16} \pm 0 \%\right)$ and for Stage $5\left(3.5 \times 10^{19} \pm 0 \%\right)$. Not only the duration of Stage 4 and Stage 5 but also the topology of the Bump 4 
located between these stages varies per Decision Difficulty (Figure 7). This Bump is characterized by higher amplitude for Easy conditions with most prominent central positivity.

To summarize, for all conditions, an HsMM-MVPA model with five stages accounted best for the data. For the Easy conditions, the HsMM-MVPA analysis demonstrated that Bump 2 and the duration of Stage 3 varied by Stimulus Type while the other stages were shared. For the Difficult conditions, no stages were found to vary by Stimulus Type. When comparing Easy and Difficult conditions, the duration of Stage 4 and Stage 5 and the topology of Bump 4 located between them were found to vary by Decision Difficulty.

Table 3. The results of model comparison for the estimated HsMM-MVPA models. The reported values express the number of participants for whom the model improved (row compared to column). The numbers in the grey background indicate a significantly better model prediction (as determined by a sign test, $\mathrm{p}<0.05$ ). Additionally, we marked the best models for Easy and Difficult conditions with asterisks (*).

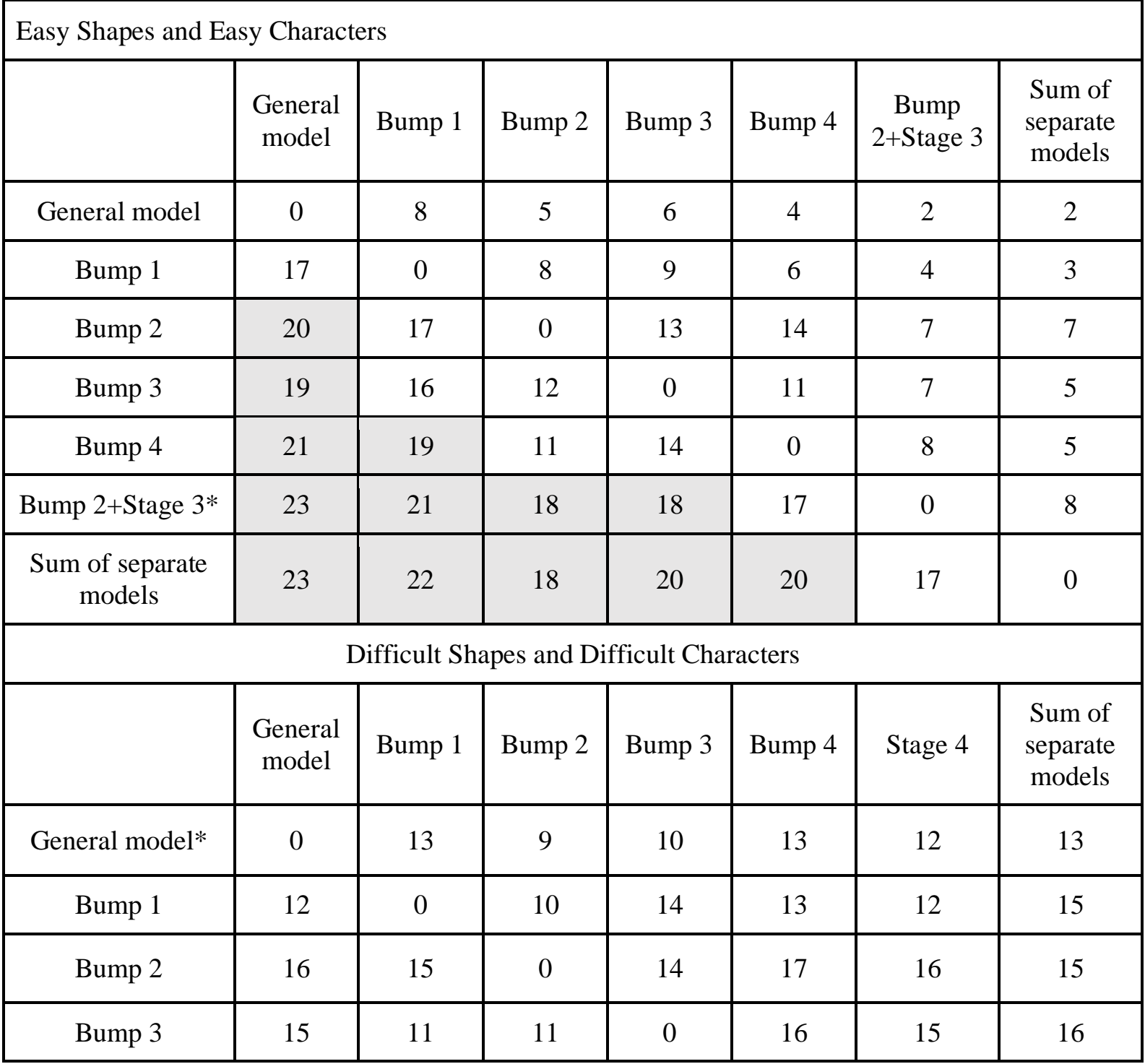




\begin{tabular}{|c|c|c|c|c|c|c|c|}
\hline Bump 4 & 12 & 12 & 8 & 9 & 0 & 6 & 11 \\
\hline Stage 4 & 13 & 13 & 9 & 10 & 19 & 0 & 14 \\
\hline $\begin{array}{c}\text { Sum of separate } \\
\text { models }\end{array}$ & 12 & 10 & 10 & 9 & 14 & 11 & 0 \\
\hline
\end{tabular}

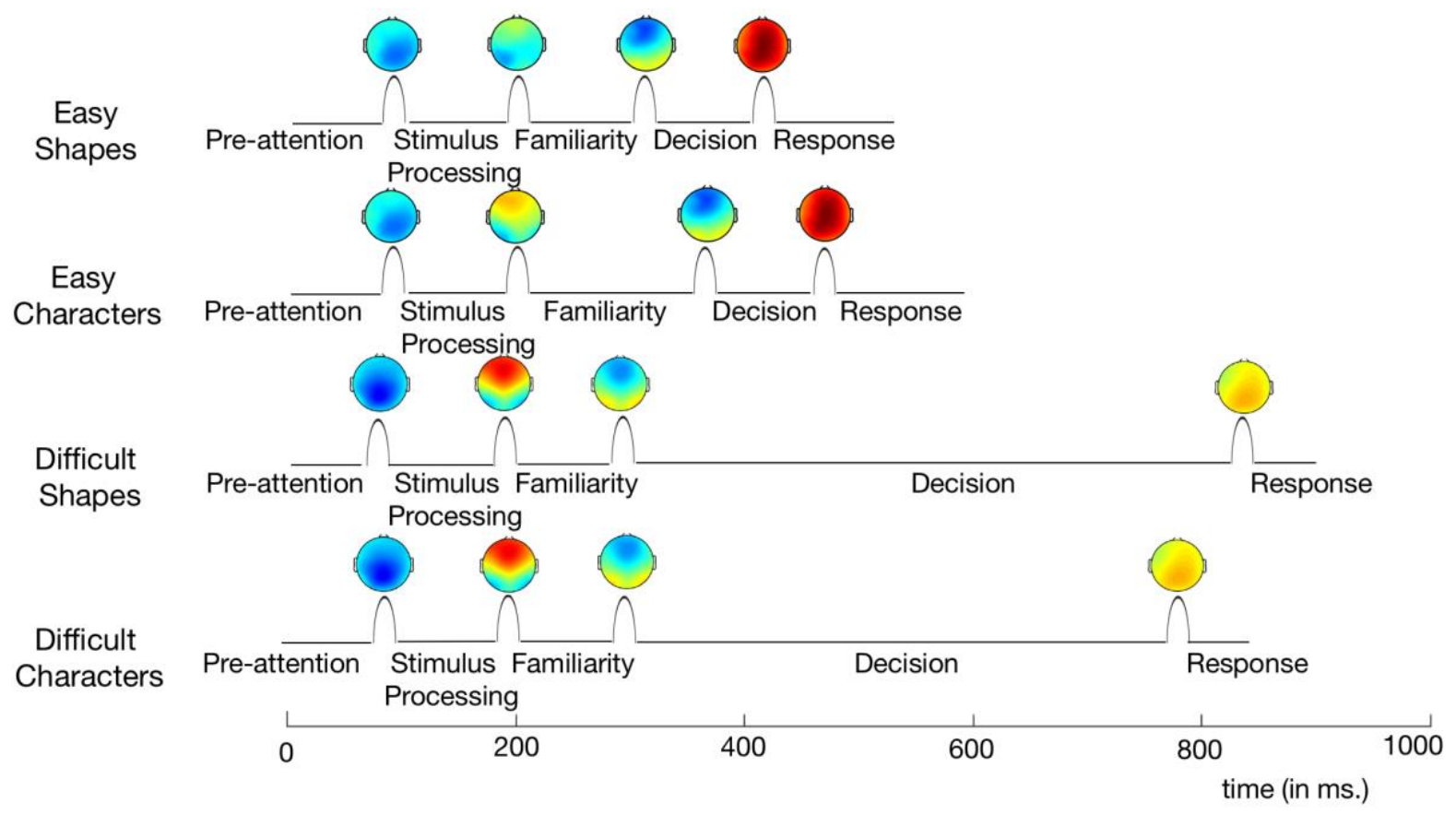

Figure 6. The topographical representations and temporal locations of the resulting HsMM-MVPA stages plotted per condition. Our cognitive interpretation of these stages is described in the Functional Interpretation of HsMMMVPA stages section.

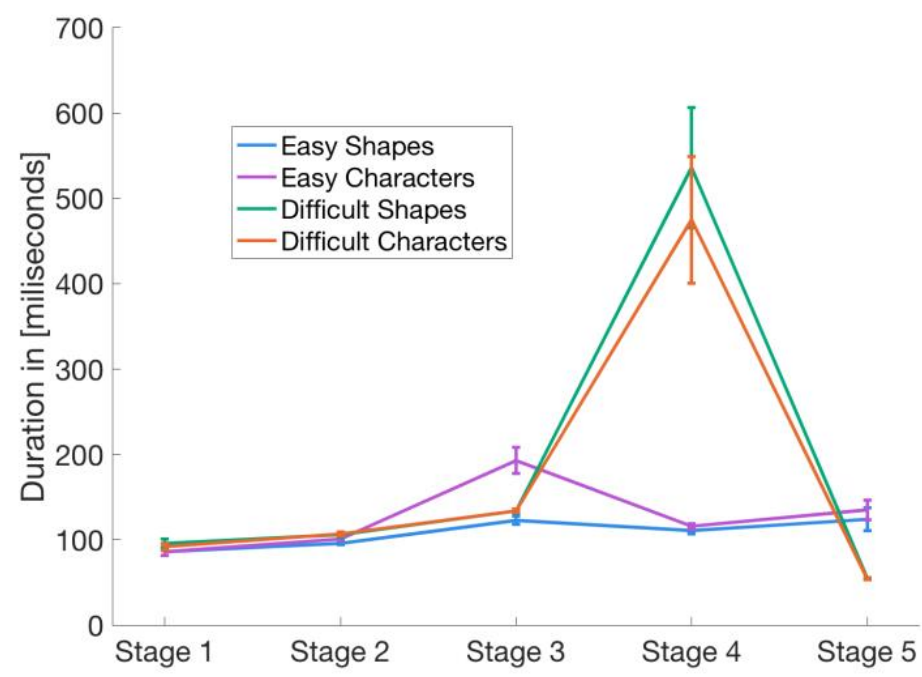

Figure 7. The average stage durations with standard errors per condition from the HsMM-MVPA models. 
We hypothesized that the stages discovered with the HsMM-MVPA method should be consistent with the results of the EAMs. To this end, we compared the duration of processing stages of the HsMM-MVPA models with the results of the Wald models. We assume that the HsMM-MVPA method decomposes the Wald decision time (based on drift rate and threshold parameters) and nondecision time into a more detailed sequence of processing stages. This suggests that a particular sum of stage durations in the HsMM-MVPA model should correspond to either the Wald decision or nondecision durations. If this assumption holds, a regression model fitted to the corresponding durations in HsMM-MVPA and Wald should have a slope not different from 1.

To test this assumption, we first computed the duration of the decision process in Wald model by dividing the threshold value $(B)$ by drift rate $(v)$. The non-decision time is simply given by the nondecision parameter $t 0$. Next, linear regression models with an intercept of 0 were fitted to the durations. In these models, Wald decision or non-decision time acted as the dependent variable whereas various HsMM-MVPA stages and their combinations were used as predictors. If the slope value for these models is not different from 1 , it can be inferred that the methods produce the same values.

For the Wald decision time, various regression models were constructed with a single HsMMMVPA stage or a sum of consecutive stages as predictors (for an overview of the values from all estimated models, see Table 4). To test which of these stages represent the Wald decision time, the resulting models were compared to a restricted model where the slope value is 1 . Next, the $F$-statistic and corresponding $p$-values were computed for these models. The model with the combined duration of the HsMM-MVPA Stages 3, 4, and 5 was the only model with a slope that was not different from 1 ( $p$ $>0.05)$. Figure $8 \mathrm{~A}$ illustrates the clear relationship between the duration of these stages and the duration of Wald decision time.

The Wald non-decision time should logically be represented in the remaining two HsMMMVPA stages: Stage 1 and 2. To test this assumption, various regression models were fitted for Wald non-decision time (Table 4). In line with our hypothesis, the regression model with the combined duration of HsMM-MVPA Stages 1, 2 was the only model with a slope not different from $1(p>0.05)$. The Wald non-decision time along with cumulative first two stages from HsMM-MVPA are represented 
in Figure 8B. It should be noted that while the two methods produce similar findings for the duration of non-decision, in the Wald models these processes have a larger variation between participants.

To summarize, there is a clear correspondence between the decision times from the two methods, where the decision time of the EAMs corresponded to the last three stages of the HsMMMVPA. Furthermore, the non-decision time for the EAMs corresponded to the first two stages.

Table 4. The slope values for the regression models with Wald durations predicted by HsMM-MVPA stages. These values are ordered by their absolute difference from a slope of 1 (starting from the model with the slope closest to 1$)$. The values significantly not different from $1(\mathrm{p}>0.05)$ are marked with asterisks $(*)$.

\begin{tabular}{|c|c|c|c|}
\hline \multicolumn{2}{|c|}{ Wald decision time as a response variable } & \multicolumn{2}{|c|}{$\begin{array}{l}\text { Wald non- decision time as a } \\
\text { response variable }\end{array}$} \\
\hline Predictors & $\begin{array}{l}\text { Slope } \\
\text { value }\end{array}$ & Predictors & $\begin{array}{l}\text { Slope } \\
\text { value }\end{array}$ \\
\hline Stage $3+$ Stage $4+$ Stage 5 & $0.983^{*}$ & Stage $1+$ Stage 2 & $1.032 *$ \\
\hline Stage $3+$ Stage 4 & 1.114 & Stage $2+$ Stage 3 & 0.796 \\
\hline Stage $2+$ Stage $3+$ Stage $4+$ Stage 5 & 0.845 & Stage $1+$ Stage $2+$ Stage 5 & 0.688 \\
\hline Stage $1+$ Stage $2+$ Stage $3+$ Stage 4 & 0.837 & Stage 3 & 1.315 \\
\hline Stage $4+$ Stage 5 & 1.25 & Stage $1+$ Stage $2+$ Stage 3 & 0.588 \\
\hline Stage 4 & 1.384 & Stage 4 & 0.383 \\
\hline Stage $1+$ Stage $2+$ Stage 3 & 1.58 & Stage $4+$ Stage 5 & 0.374 \\
\hline Stage $2+$ Stage 3 & 2.114 & Stage $3+$ Stage 4 & 0.34 \\
\hline Stage $1+$ Stage 2 & 2.843 & Stage $3+$ Stage $4+$ Stage 5 & 0.313 \\
\hline Stage 3 & 3.422 & $\begin{array}{c}\text { Stage } 2+\text { Stage } 3+\text { Stage } 4+ \\
\text { Stage } 5\end{array}$ & 0.274 \\
\hline Stage 5 & 4.247 & $\begin{array}{c}\text { Stage } 1+\text { Stage } 2+\text { Stage } 3+ \\
\text { Stage } 4\end{array}$ & 0.268 \\
\hline Stage 2 & 5.3 & Stage 5 & 1.743 \\
\hline Stage 1 & 6.115 & Stage 1 & 2.2 \\
\hline
\end{tabular}



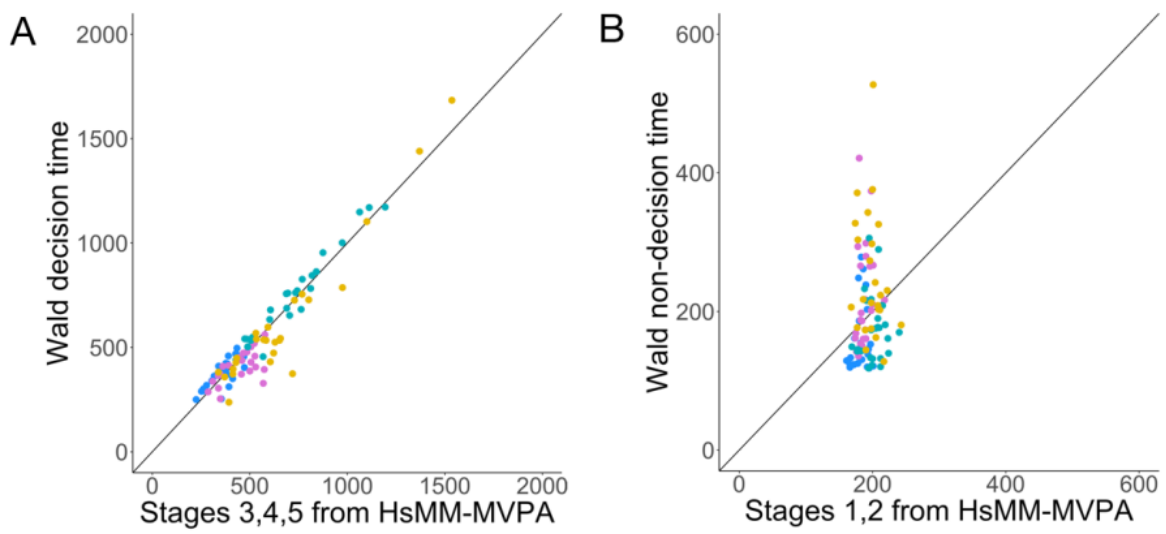

Easy Shapes

Easy Characters

Difficult Shapes

Difficult Characters

Figure 8. Comparison of the durations from HsMM-MVPA analysis and Wald modelling. The points in the figure indicate the values for each participant and condition and are supplemented by a diagonal line.

\section{Functional interpretation of HsMM-MVPA stages}

In all four conditions, we found evidence for the existence of five cognitive stages. The next step is to interpret those stages in terms of the underlying cognitive processes. The EAMs suggest that stages 1-2 are perceptual, while stages 3-5 concern decision making. Here, we will review the literature to obtain independent evidence for such a functional interpretation of the discovered stages.

The first bump of all conditions has an average onset of around $100 \mathrm{~ms}$ and is characterized by central-parietal negativity (Figure 6). These findings resemble a standard ERP N1 component that has been largely associated with attention (Luck, 2005; Luck, Woodman, \& Vogel, 2000). Stage 1 is, therefore, most likely a pre-attention stage (cf. Anderson et al., 2016; Zhang, Walsh, et al., 2017, 2018). While Bump 2 was found to be different between Easy Shapes and Easy Characters, on close inspection, it appears that this bump has very similar topological distributions in all conditions, with different amplitudes. With its average onset of around $200 \mathrm{~ms}$ and prominent frontal positivity (most salient for the Difficult conditions), the bump resembles a P2 component. Since its discovery, this component has been largely associated with attention (e.g., Miltner, Johnson, Braun, \& Larbig, 1989; Rugg, Milner, Lines, \& Phalp, 1987) and more recently with initial word processing (Lee, Liu, \& Tsai, 2012; Mangels, Picton, \& Craik, 2001). This provides evidence for the interpretation of Stage 2 as the Stimulus Processing stage. Taken together, this fits the interpretation of these stages as non-decision stages of the EAMs. 
In its frontocentral negativity and average onset, Bump 3 is similar to the FN400 component that was has been widely accepted to refer to familiarity-driven recognition (Curran, 2000; Paller, Voss, \& Boehm, 2007; Rugg et al., 1998). In an alternative approach, this frontal negativity effect was linked to a decisional account (Dobbins \& Han, 2006; Hayama, Johnson, \& Rugg, 2008). The frontal negativity and average onset of Stage 3 are compatible with both interpretations. Thus, if we accept the familiarity interpretation of the frontal negative component, we would assume that during this stage the participants were recognizing the patterns in the stimuli (e.g., shape or color). If we accept the decisional interpretation, we would assume that this activity arises from a number of internal decisions participants had to make. The current findings cannot arbitrate between these different interpretations; however, given the current task, we tentatively propose the interpretation of this stage as Familiarity Stage.

In both the Shapes and Characters conditions, the last two stages (Stage 4 and Stage 5) were found to vary in their duration by Decision Difficulty. The largest difference was represented in Stage 4 - being significantly longer when the decision was more difficult. Additionally, Bump 4 that terminated this stage had a different topology with a more positive-going waveform across the scalp when the decision was easier. We, therefore, interpret Stage 4 as the core decision making stage in which the details of the stimulus are recognized and mapped onto a response. The last stage then represents Response execution. The differences in the duration of this stage were also statistically significant albeit in the opposite direction: being significantly shorter when the decision was more difficult. As response mapping for Easy and Difficult conditions was identical, we assume that participants started preparing the response execution while making a decision in Difficult conditions (Stage 4), leading to a shorter response execution stage. Taken together, this fits the interpretation of the last three stages (Stage 3, Stage 4, Stage 5) as decision stages of the Wald model, where we should note that decision making includes motor execution.

\section{General Discussion}

The goal of the current paper was to establish evidence for HsMM-MVPA analysis (Anderson et al., 2016) as a method capable of parsing a cognitive task into meaningful stages. This question was addressed by designing four conditions that varied in perceptual processing and decision difficulty and 
complementing the HsMM-MVPA analysis with EAMs. EAMs provide insights into cognitive processing by decomposing reaction times into decision and non-decision processes. In turn, HsMMMVPA method allows us to zoom into these processes by finding a range of underlying processing stages. The simplicity of the designed tasks, the experimental manipulations, and the information obtained employing EAMs, allowed us to test whether the detection of the stages and their cognitive interpretation is valid.

First, we demonstrated that the manipulations in Stimulus Type and Decision Difficulty led to significant differences in behavior, with the largest differences in reaction times when a decision was more difficult. In addition, in the Easy conditions participants were slower to respond to Characters than to Shapes. Second, to quantify the duration of decision and non-decision components, reaction times were decomposed with EAMs. We found two main effects: non-decision time was longer for Characters than for Shapes, and drift rate was higher for Easy decisions than for Difficult decisions. This matches previous studies that demonstrated that lower drift rate values express difficulty in the decision (e.g., Basten et al., 2010; Donkin \& Van Maanen, 2014; Turner, Van Maanen, \& Forstmann, 2015). Specifically, some studies that manipulate decision difficulty by increasing the set of response alternatives found that this led to lower drift rates (Anders, Riès, van Maanen, \& Alario, 2015; van Maanen et al., 2012). This manipulation seems comparable to our experimental design, where the number of stimulus-response mappings varied between easy and difficult choices.

To discover the processing stages underlying these components, the HsMM-MVPA analysis was applied. For all conditions, a model with five stages accounted best for the data. We found two main effects: in Easy Conditions the topology of Bump 2 and the duration of the consecutive stage varied by Stimulus Type, and the topology and the duration of the last two stages varied by Decision Difficulty. The largest difference in Decision Difficulty was represented in Stage 4, which was significantly longer when decisions were more difficult.

The experimental manipulations in Stimulus Type and Decision Difficulty were reflected in both HsMM-MVPA and EAMs. While EAMs allowed us to quantitatively assess the effects of these manipulations by decomposing reaction times, HsMM-MVPA uncovered the cognitive stages that cause these differences. To explore the relationship between the results of the HsMM-MVPA method and the 
EAMs in more detail, we compared their respective decision and non-decision durations. There was a clear consistency between the methods: we found an almost perfect correspondence between the duration of stages 3-5 of the HsMM-MVPA method and the decision time of the EAMs. Not only was this effect present on the means, but both methods captured the variability in decision durations for each condition and participant in a similar manner. Based on the literature, these HsMM-MVPA stages were interpreted as Familiarity, Decision and Response. Note that this means that motor execution is part of the decision process estimated by the EAMs. Although motor processes are typically thought to be part of the non-decision time, there is increasing evidence that motor (preparation) is part of the decision process itself (e.g., Spieser, Servant, Hasbroucq, \& Burle, 2017; Thura \& Cisek, 2014, 2016).

Consequently, the non-decision component in EAMs was represented in the first two HsMMMVPA stages. However, here the connection was less strong: the EAMs reported a much higher variance of these stages than the HsMM-MVPA method (Figure 8), and while the EAMs showed a difference in non-decision time between Stimulus Types, this difference was not present in the HsMMMVPA stages. If one assumes that the conclusions of the HsMM-MVPA approach are correct, one would conclude that EAMs overestimate the variability in non-decision time. One may suspect that this is the case since the reaction times that are being modelled do not only reflect underlying cognitive processes but are influenced by alternative processes such as motor planning (Haith, Pakpoor, \& Krakauer, 2016; Walsh et al., 2017). Additionally, recent studies demonstrated that reaction times measures are biased and context-dependent (Wong, Goldsmith, Forrence, Haith, \& Krakauer, 2017; Wong, Goldsmith, \& Krakauer, 2016). Because the EAMs that we used here are fully dependent on the RT measures, this variation is spread to all stages. However, the HsMM-MVPA method uses the ongoing EEG and can attribute this variation to the actual stages that are influenced. In terms of cognitive interpretation, this might mean that the early non-decision processes such as attention and stimulus encoding are biologically defined mechanisms that are very stable across different tasks and participants - early ERP components are also very stable typically - while the decision stages are more contextdependent. Alternatively, it might also be that the HsMM-MVPA analysis underestimates the variation in non-decision time duration and attributes all variability to the stages that are linked strongest to overall 
RT (cf. Walsh et al., 2017). However, given the very small standard errors on the duration of the early perceptual stages, we think this explanation is less likely.

\section{Conclusion}

With this study, we have demonstrated the value of the HsMM-MVPA analysis as a method to detect detailed cognitive stages in a task. On a more general level, the combination of the HsMM-MVPA models and EAMs allowed us to robustly answer the question What are the stages people go through when they perform simple discrimination tasks? The EAMs helped us to quantify the decision and nondecision components underlying the task performance, while the HsMM-MVPA method provided us with a detailed division of these components into processing stages. Numerous theories have modelled the performance on different tasks starting from perceptual processing to response execution with a topdown strategy. We suggest that HsMM-MVPA analysis can inform the development of such process models as it derives the stages directly from EEG data in the opposite, bottom-up manner.

Furthermore, we would like to suggest that the analysis of simple tasks can be potentially used to interpret more complex tasks that require the involvement of higher cognitive processes. At present, a range of complex tasks was analyzed with the HsMM-MVPA method applied to EEG. These tasks included oddball paradigm tasks (Walsh et al., 2017), associative recognition (Anderson et al., 2016), visual working memory task (Zhang, van Vugt, et al., 2018) and others. Although in each of these tasks certain cognitive stages were detected, it remains difficult to integrate these findings and to create basic profiles of cognitive stages. While the stages we discovered might be specific to our tasks, given how similar these stage definitions are to previously published stages (e.g., Anderson et al., 2016), it seems likely that they can be used as the starting point for creating a library of neural signatures associated with certain cognitive processes. 


\section{Acknowledgments}

This research was supported by Netherlands Organisation for Scientific Research (NWO) Veni grant 451-15-040 awarded to Jelmer Borst. Van Rijn was supported by the research programme "Interval Timing in the Real World: A functional, computational and neuroscience approach", project number 453-16-005, financed by the Netherlands Organisation for Scientific Research (NWO).

The authors want to thank Daan Sijbring for helping in data collection for Experiment 1, Andrew Heathcote and Steven Miletic for their help and feedback on Wald modelling during Modelbased Brainhack in Amsterdam, Jacolien van Rij for her feedback on LME analysis.

\section{Data sharing}

All the data for this publication is available at https://osf.io/653ec/

\section{References}

Anders, R., Riès, S., van Maanen, L., \& Alario, F. X. (2015). Evidence accumulation as a model for lexical selection. Cognitive Psychology. https://doi.org/10.1016/j.cogpsych.2015.07.002

Anders, R., Alario, F. X., \& Maanen, L. Van. (2016). The shifted wald distribution for response time data analysis. Psychological Methods. https://doi.org/10.1037/met0000066

Anderson, J. R., Borst, J. P., Fincham, J. M., Ghuman, A. S., Tenison, C., \& Zhang, Q. (2018). The Common Time Course of Memory Processes Revealed. Psychological Science. https://doi.org/10.1177/0956797618774526

Anderson, J. R., Zhang, Q., Borst, J. P., \& Walsh, M. M. (2016). The discovery of processing stages: Extension of Sternberg's method. Psychological Review. https://doi.org/10.1037/rev0000030

Barr, D. J., Levy, R., Scheepers, C., \& Tily, H. J. (2013). Random effects structure for confirmatory hypothesis testing: Keep it maximal. Journal of Memory and Language. https://doi.org/10.1016/j.jml.2012.11.001

Basten, U., Biele, G., Heekeren, H. R., \& Fiebach, C. J. (2010). How the brain integrates costs and benefits during decision making. Proceedings of the National Academy of Sciences of the United 
States of America. https://doi.org/10.1073/pnas.0908104107

Bates, D. M., \& DebRoy, S. (2004). Linear mixed models and penalized least squares. Journal of Multivariate Analysis. https://doi.org/10.1016/j.jmva.2004.04.013

Bates, D. M., Kliegl, R., Vasishth, S., \& Baayen, H. (2015). Parsimonious mixed models Douglas Bates. ArXiv E-Print, under Revision.

Bell, A. J., \& Sejnowski, T. J. (1995). An information-maximization approach to blind separation and blind deconvolution. Neural Computation.

Bode, S., Bennett, D., Sewell, D. K., Paton, B., Egan, G. F., Smith, P. L., \& Murawski, C. (2018). Dissociating neural variability related to stimulus quality and response times in perceptual decision-making. Neuropsychologia. https://doi.org/10.1016/j.neuropsychologia.2018.01.040

Boehm, U., Van Maanen, L., Forstmann, B., \& Van Rijn, H. (2014). Trial-by-trial fluctuations in CNV amplitude reflect anticipatory adjustment of response caution. NeuroImage. https://doi.org/10.1016/j.neuroimage.2014.03.063

Bogacz, R., Wagenmakers, E. J., Forstmann, B. U., \& Nieuwenhuis, S. (2010). The neural basis of the speed-accuracy tradeoff. Trends in Neurosciences. https://doi.org/10.1016/j.tins.2009.09.002

Borst, J. P., \& Anderson, J. R. (2015). The discovery of processing stages: Analyzing EEG data with hidden semi-Markov models. NeuroImage. https://doi.org/10.1016/j.neuroimage.2014.12.029

Cohen, X. M. (2014). Analyzing Neural Time Series Data. MIT Press. https://doi.org/10.1007/s13398014-0173-7.2

Curran, T. (2000). Brain potentials of recollection and familiarity. Memory and Cognition. https://doi.org/10.3758/BF03209340

Delorme, A., \& Makeig, S. (2004). EEGLAB: An open source toolbox for analysis of single-trial EEG dynamics including independent component analysis. Journal of Neuroscience Methods. https://doi.org/10.1016/j.jneumeth.2003.10.009

Dobbins, I. G., \& Han, S. (2006). Isolating rule- versus evidence-based prefrontal activity during episodic and lexical discrimination: A functional magnetic resonance imaging investigation of detection theory distinctions. Cerebral Cortex. https://doi.org/10.1093/cercor/bhj098

Donders, F. C. (1868|1969). On the speed of mental processes. Acta Psychologica. 
https://doi.org/10.1016/0001-6918(69)90065-1

Donkin, C., \& Van Maanen, L. (2014). Piéron's Law is not just an artifact of the response mechanism. Journal of Mathematical Psychology. https://doi.org/10.1016/j.jmp.2014.09.006

Evans, N. J., \& Wagenmakers, E.-J. (2020). Evidence Accumulation Models: Current Limitations and Future Directions. The Quantitative Methods for Psychology. https://doi.org/10.20982/tqmp.16.2.p073

Gelman, A., \& Rubin, D. B. (1992). Inference from iterative simulation using multiple sequences. Statistical Science. https://doi.org/10.1214/ss/1177011136

Goldstein, E. B., Humphreys, G. W., Shiffrar, M., \& Yost, W. A. (2008). Blackwell Handbook of Sensation and Perception. Blackwell Handbook of Sensation and Perception. https://doi.org/10.1002/9780470753477

Gomez, P., Ratcliff, R., \& Perea, M. (2007). A Model of the Go/No-Go Task. Journal of Experimental Psychology: General. https://doi.org/10.1037/0096-3445.136.3.389

Haith, A. M., Pakpoor, J., \& Krakauer, J. W. (2016). Independence of movement preparation and movement initiation. Journal of Neuroscience. https://doi.org/10.1523/JNEUROSCI.324515.2016

Hayama, H. R., Johnson, J. D., \& Rugg, M. D. (2008). The relationship between the right frontal old/new ERP effect and post-retrieval monitoring: Specific or non-specific? Neuropsychologia. https://doi.org/10.1016/j.neuropsychologia.2007.11.021

Heathcote, A. (2004). Fitting Wald and ex-Wald distributions to response time data: An example using functions for the S-PLUS package. Behavior Research Methods, Instruments, and Computers. https://doi.org/10.3758/BF03206550

Heathcote, A., Lin, Y.-S., Reynolds, A., Strickland, L., Gretton, M., \& Matzke, D. (2019). Dynamic models of choice. Behavior Research Methods, 51(2), 961-985. https://doi.org/10.3758/s13428018-1067-y

Henson, R. N. (2011). How to discover modules in mind and brain: The curse of nonlinearity, and blessing of neuroimaging. A comment on Sternberg (2011). Cognitive Neuropsychology. https://doi.org/10.1080/02643294.2011.561305 
Hoeting, J. A., Madigan, D., Raftery, A. E., \& Volinsky, C. T. (1999). Bayesian model averaging: A tutorial. Statistical Science. https://doi.org/10.1214/ss/1009212519

Hurvich, L. M., \& Jameson, D. (1957). An opponent-process theory of color vision. Psychological Review. https://doi.org/10.1037/h0041403

Jepma, M., Wagenmakers, E. J., \& Nieuwenhuis, S. (2012). Temporal expectation and information processing: A model-based analysis. Cognition. https://doi.org/10.1016/j.cognition.2011.11.014

Kass, R. E., \& Raftery, A. E. (1995). Bayes factors. Journal of the American Statistical Association. https://doi.org/10.1080/01621459.1995.10476572

Klimesch, W., Sauseng, P., \& Hanslmayr, S. (2007). EEG alpha oscillations: The inhibition-timing hypothesis. Brain Research Reviews. https://doi.org/10.1016/j.brainresrev.2006.06.003

Kuznetsova, A., Brockhoff, P. B., \& Christensen, R. H. B. (2018). lmerTest Package: Tests in Linear Mixed Effects Models. R package version 3.0-1. Journal of Statistical Software. https://doi.org/10.18637/jss.v082.i13

Lee, C. Y., Liu, Y. N., \& Tsai, J. L. (2012). The time course of contextual effects on visual word recognition. Frontiers in Psychology. https://doi.org/10.3389/fpsyg.2012.00285

Luck, S. J. (2005). An Introduction to the Event-Related Potential Technique. Monographs of the Society for Research in Child Development. https://doi.org/10.1118/1.4736938

Luck, S. J., Woodman, G. F., \& Vogel, E. K. (2000). Event-related potential studies of attention. Trends in Cognitive Sciences. https://doi.org/10.1016/S1364-6613(00)01545-X

Makeig, S., Westerfield, M., Jung, T. P., Enghoff, S., Townsend, J., Courchesne, E., \& Sejnowski, T. J. (2002). Dynamic brain sources of visual evoked responses. Science. https://doi.org/10.1126/science.1066168

Mangels, J. A., Picton, T. W., \& Craik, F. I. M. (2001). Attention and successful episodic encoding: An event-related potential study. Cognitive Brain Research. https://doi.org/10.1016/S09266410(00)00066-5

Matzke, D., \& Wagenmakers, E. J. (2009). Psychological interpretation of the ex-gaussian and shifted wald parameters: A diffusion model analysis. Psychonomic Bulletin and Review. https://doi.org/10.3758/PBR.16.5.798 
Miletić, S., \& van Maanen, L. (2019). Caution in decision-making under time pressure is mediated by timing ability. Cognitive Psychology. https://doi.org/10.1016/j.cogpsych.2019.01.002

Milosavljevic, M., Malmaud, J., Huth, A., Koch, C., \& Rangel, A. (2010). The Drift Diffusion Model can account for the accuracy and reaction time of value-based choices under high and low time pressure. Judgment and Decision Making. https://doi.org/10.2139/ssrn.1901533

Miltner, W., Johnson, R., Braun, C., \& Larbig, W. (1989). Somatosensory event-related potentials to painful and non-painful stimuli: effects of attention. Pain. https://doi.org/10.1016/0304$3959(89) 90217-0$

Morey, R. D. (2008). Confidence Intervals from Normalized Data: A correction to Cousineau (2005). Tutorials in Quantitative Methods for Psychology. https://doi.org/10.20982/tqmp.04.2.p061

Morey, R. D., Rouder, J. N., \& Jamil, T. (2015). Package ' BayesFactor .' R Package Version 0.9.122.

Mulder, M. J., van Maanen, L., \& Forstmann, B. U. (2014). Perceptual decision neurosciences - a model-based review. Neuroscience. https://doi.org/10.1016/j.neuroscience.2014.07.031

Paller, K. A., Voss, J. L., \& Boehm, S. G. (2007). Validating neural correlates of familiarity. Trends in Cognitive Sciences. https://doi.org/10.1016/j.tics.2007.04.002

Portoles, O., Borst, J. P., \& van Vugt, M. K. (2018). Characterizing synchrony patterns across cognitive task stages of associative recognition memory. European Journal of Neuroscience. https://doi.org/10.1111/ejn.13817

Posner, M. I. (2005). Timing the brain: Mental chronometry as a tool in neuroscience. PLoS Biology. https://doi.org/10.1371/journal.pbio.0030051

Ratcliff, R. (1978). A theory of memory retrieval. Psychological Review. https://doi.org/10.1037/0033-295X.85.2.59

Ratcliff, R., \& McKoon, G. (2008). The diffusion decision model: Theory and data for two-choice decision tasks. Neural Computation. https://doi.org/10.1162/neco.2008.12-06-420

Rugg, M. D., Milner, A. D., Lines, C. R., \& Phalp, R. (1987). Modulation of visual event-related potentials by spatial and non-spatial visual selective attention. Neuropsychologia. https://doi.org/10.1016/0028-3932(87)90045-5 
Rugg, M. D., Mark, R. E., Walla, P., Schloerscheidt, A. M., Birch, C. S., \& Allan, K. (1998).

Dissociation of the neural correlates of implicit and explicit memory. Nature. https://doi.org/10.1038/33396

Schroeder, C. E., Steinschneider, M., Javitt, D. C., Tenke, C. E., Givre, S. J., Mehta, A. D., ... Vaughan, H. G. (1995). Localization of ERP generators and identification of underlying neural processes. Electroencephalography and Clinical Neurophysiology. Supplement.

Shah, A. S., Bressler, S. L., Knuth, K. H., Ding, M., Mehta, A. D., Ulbert, I., \& Schroeder, C. E. (2004). Neural dynamics and the fundamental mechanisms of event-related brain potentials. Cerebral Cortex (New York, N.Y. : 1991). https://doi.org/10.1093/cercor/bhh009

Sherri, M., Boulkaibet, I., Marwala, T., \& Friswell, M. I. (2019). A Differential Evolution Markov Chain Monte Carlo Algorithm for Bayesian Model Updating. https://doi.org/10.1007/978-3-31975390-4_9

Smith, P. L., \& Ratcliff, R. (2004). Psychology and neurobiology of simple decisions. Trends in Neurosciences. https://doi.org/10.1016/j.tins.2004.01.006

Spieser, L., Servant, M., Hasbroucq, T., \& Burle, B. (2017). Beyond decision! Motor contribution to speed-accuracy trade-off in decision-making. Psychonomic Bulletin and Review. https://doi.org/10.3758/s13423-016-1172-9

Sternberg, S. (1969). The discovery of processing stages: Extensions of Donders' method. Acta Psychologica. https://doi.org/10.1016/0001-6918(69)90055-9

Thura, D., \& Cisek, P. (2014). Deliberation and commitment in the premotor and primary motor cortex during dynamic decision making. Neuron. https://doi.org/10.1016/j.neuron.2014.01.031

Thura, D., \& Cisek, P. (2016). Modulation of premotor and primary motor cortical activity during volitional adjustments of speed-accuracy trade-offs. Journal of Neuroscience. https://doi.org/10.1523/JNEUROSCI.2230-15.2016

Turner, B. M., Sederberg, P. B., Brown, S. D., \& Steyvers, M. (2013). A Method for efficiently sampling from distributions with correlated dimensions. Psychological Methods. https://doi.org/10.1037/a0032222

Turner, B. M., Van Maanen, L., \& Forstmann, B. U. (2015). Informing cognitive abstractions through 
neuroimaging: The neural drift diffusion model. Psychological Review.

https://doi.org/10.1037/a0038894

van Maanen, L., Grasman, R. P. P. P., Forstmann, B. U., Keuken, M. C., Brown, S. D., \& Wagenmakers, E. J. (2012). Similarity and number of alternatives in the random-dot motion paradigm. Attention, Perception, and Psychophysics. https://doi.org/10.3758/s13414-011-0267-7 van Maanen, L., van der Mijn, R., van Beurden, M. H. P. H., Roijendijk, L. M. M., Kingma, B. R. M., Miletić, S., \& van Rijn, H. (2019). Core body temperature speeds up temporal processing and choice behavior under deadlines. Scientific Reports. https://doi.org/10.1038/s41598-019-46073-3

Vehtari, A., Gelman, A., \& Gabry, J. (2015). Efficient implementation of leav-one-out crossvalidation and WAIC for evaluating fitted Bayesian models. ArXiv. https://doi.org/10.1007/s11222-016-9696-4

Wagenmakers, E. J., \& Farrell, S. (2004). AIC model selection using Akaike weights. Psychonomic Bulletin and Review. https://doi.org/10.3758/BF03206482

Walsh, M. M., Gunzelmann, G., \& Anderson, J. R. (2017). Relationship of P3b single-trial latencies and response times in one, two, and three-stimulus oddball tasks. Biological Psychology. https://doi.org/10.1016/j.biopsycho.2016.11.011

Wong, A. L., Goldsmith, J., Forrence, A. D., Haith, A. M., \& Krakauer, J. W. (2017). Reaction times can reflect habits rather than computations. ELife. https://doi.org/10.7554/eLife.28075

Wong, A. L., Goldsmith, J., \& Krakauer, J. W. (2016). A motor planning stage represents the shape of upcoming movement trajectories. Journal of Neurophysiology. https://doi.org/10.1152/jn.01064.2015

Wu, C. F. J. (1983). On the Convergence Properties of the EM Algorithm. The Annals of Statistics. https://doi.org/10.1214/aos/1176346060

Yeung, N., Bogacz, R., Holroyd, C. B., \& Cohen, J. D. (2004). Detection of synchronized oscillations in the electroencephalogram: An evaluation of methods. Psychophysiology. https://doi.org/10.1111/j.1469-8986.2004.00239.x

Yu, S. Z. (2010). Hidden semi-Markov models. Artificial Intelligence. https://doi.org/10.1016/j.artint.2009.11.011 
Zhang, Q., Borst, J. P., Kass, R. E., \& Anderson, J. R. (2017). Inter-subject alignment of MEG datasets in a common representational space. Human Brain Mapping. https://doi.org/10.1002/hbm.23689

Zhang, Q., van Vugt, M., Borst, J. P., \& Anderson, J. R. (2018). Mapping working memory retrieval in space and in time: A combined electroencephalography and electrocorticography approach. NeuroImage. https://doi.org/10.1016/j.neuroimage.2018.03.039

Zhang, Q., Walsh, M. M., \& Anderson, J. R. (2017). The effects of probe similarity on retrieval and comparison processes in associative recognition. Journal of Cognitive Neuroscience. https://doi.org/10.1162/jocn_a_01059

Zhang, Q., Walsh, M. M., \& Anderson, J. R. (2018). The Impact of Inserting an Additional Mental Process. Computational Brain \& Behavior. https://doi.org/10.1007/s42113-018-0002-8 